\title{
MONTE CARLO MODELING OF THE PHOTON-ELECTRON CASCADE IN HETEROGENEOUS MATTER
}

\author{
MIKHAIL B. MARKOV, ROMAN V. USKOV ${ }^{*}$, MIKHAIL E. ZHUKOVSKIY \\ Keldysh Institute of Applied Mathematics of RAS (KIAM), \\ Miusskaya sq. 4, 125047, Moscow, Russia \\ *Corresponding author. E-mail: roman.uskov@gmail.com, web page: http://keldysh.ru
}

DOI: 10.20948/mathmontis-2019-46-5

Summary. Algorithms of statistical simulating the processes of the photon-electron cascade transport in objects using heterogeneous computers is worked out. The processes include the generation of electron fluxes resulting in photon collisions, the appearance of photons due to bremsstrahlung and pair production. The cascade tree processing method is developed. It considers significant difference in physical properties of different particles and allows constructing the particle trajectories economically. Random photon trajectories are constructed by use of ray-tracing the whole irradiated object. Electron trajectories are created in "quasi-homogeneous" region of electron stopping path size. A weight algorithm for registration of events of low probability is constructed. The algorithm is based on the splitting the photon trajectories. Modern technologies of calculation parallelization MPI and NVidia CUDA are used. Some results of the model calculations on the hybrid calculating cluster (HCC) K100 (http://www.kiam.ru/MVS/resourses/k100.html) are represented.

\section{INTRODUCTION}

Mathematical modeling is an effective and powerful means for investigating the properties of modern materials of the complex structure. It is useful for developing complex experimental methods [1 - 6], as well as investigation of effectiveness and reliability of technical equipment [7]. Investigating the process of transformation of X-ray radiation into electron flux and vice versa are of interest when creating amplifier screens or developing new X-ray equipment.

Radiation transport is a cascade process involving particles of different types. Compton scattering of gamma-radiation as well as photoelectric absorption and pair production leads to emergence of rapid electrons. Rapid electrons can generate additional electrons as the result of impact ionization and photons as the result of bremsstrahlung or electron-positron annihilation. The cascade modelling is complicated by the huge number of the particles. It is mainly the result of secondary electrons generation during impart ionisation process. Another difficulty is significant difference in physical properties of different particles like penetrating ability of the photons and electrons. These abilities may differ thousand times. The number of collisions along trajectory varies greatly for the photon and electron as well.

Hybrid computing clusters (HCC) development brings new possibilities of the cascade radiation transport modelling. One is the development of the fundamental model of electrons transport $[8,9]$ allowing detail research of electrons exposure to microstructure elements of the object being under radiation. The use of the hybrid architecture however requires specific approach to organising the calculations to achieve maximum GPU performance and proper

2010 Mathematics Subject Classification: 97M50, 97N50, 93A30.

Key words and Phrases: Photon-electron cascade processes, Hybrid parallelization, Monte Carlo weight algorithm. 
simultaneous CPU/GPU load [10]. Approaches for modelling of cascade processes of radiation propagation in complex objects are developed to perform calculations on heterogeneous computational equipment.

Some results of the model calculations on the hybrid calculating cluster HCC K100 (http://www.kiam.ru/MVS/resourses/k100.html) are represented.

\section{MODEL OF THE RADIATION INTERACTION WITH MATTER}

We consider processes of the interaction between radiation and matter when the time of changing the source strength is considerably longer than the lifetime of the particles in dense matter of the object being under radiation. In this case, the quasi-stationary integraldifferential equation of the photon and electron transport are true. Time is considered as a parameter. The description of the particle state uses variables $x=(\mathrm{r}, \Omega, E)$, where $\mathrm{r}, \Omega, E$ are coordinates, direction of motion and energy, respectively. The integro-differential equation for the density of the flow of particles $\Phi(x)$ is reduced to the Fredholm integral equation of the 2nd kind [9]:

$$
\begin{gathered}
\Phi(x)=\Phi_{0}(x)+\int_{0}^{\infty} d \xi \exp \{-\tau(\mathrm{r}-\xi \Omega)\} \int d \Omega^{\prime} \int d E^{\prime} \mu_{s}\left(\mathrm{r}-\xi \Omega, \Omega^{\prime}, E^{\prime}\right) \Phi\left(\mathrm{r}-\xi \Omega, \Omega^{\prime}, E^{\prime}\right), \\
\Phi_{0}(\mathbf{r}, \boldsymbol{\Omega}, E)=\int_{0}^{\infty} d \xi \exp \{-\tau(\mathbf{r}-\xi \boldsymbol{\Omega}, \mathbf{r}, E)\} S(\mathbf{r}-\xi \mathbf{\Omega}, \boldsymbol{\Omega}, E) .
\end{gathered}
$$

Formula (2) describes the flux of undistracted particles from external and internal sources of radiation; $S(x)$ is the radiation source; $\tau\left(\mathbf{r}^{\prime}, \mathbf{r}\right)=\int_{0}^{\xi} \mu\left(\mathbf{r}-\xi^{\prime} \Omega\right) d \xi^{\prime}$ is the optical distance (depth) between points $\mathbf{r}$ and $\mathbf{r}^{\prime}=\mathbf{r}-\xi \boldsymbol{\Omega} ; \mu, \mu_{s}$ are the full and differential macroscopic scattering cross sections respectively.

The complicated process of particle transport through the matter can be represented by a sequence of elementary processes of the interaction between the particle and the atoms of matter (particle trajectory). These processes include the scattering, braking or disappearance of the particle due to absorption or escape from the considered system (from the object). This representation is convenient for modelling the radiation transport by the Monte-Carlo method. In doing this, the considered transport equation is converted to the form:

$$
Q(x)=Q_{1}(x)+\int_{0}^{\infty} d \xi \cdot \exp \{-\tau(\mathbf{r}-\xi \mathbf{\Omega}, \mathbf{r}, E)\} \int d \mathbf{\Omega}^{\prime} \int d E^{\prime} \mu_{s}\left(\mathbf{r}, \mathbf{\Omega}^{\prime}, \mathbf{\Omega}, E^{\prime}, E\right) \frac{\mu(x)}{\mu\left(x^{\prime}\right)} Q\left(x^{\prime}\right) .
$$

In formula (3) variable $Q(x)=\mu(x) \Phi(x)$ is the density of collisions and $Q_{1}(x)=\mu(x) \Phi_{0}(x)$ is the density of the first collisions. The kernel of the integral operator $k=\exp \{-\tau(\mathbf{r}-\xi \mathbf{\Omega}, \mathbf{r}, E)\} \mu_{s}\left(\mathbf{r}, \mathbf{\Omega}^{\prime}, \mathbf{\Omega}, E^{\prime}, E\right) \frac{\mu(x)}{\mu\left(x^{\prime}\right)}$ has the meaning of probability density of $x^{\prime} \rightarrow x$ transition. 
The transport of the particles accompanied by the birth of secondary particles in cascade processes of the interaction of radiation with matter (for example, the birth of electrons during Compton scattering, photo-absorption of X-ray or gamma radiation, pair production) is described by a system of integral equations. The following chain can be an example of such a process: gamma-radiation-Compton and photo electrons-electrons deceleration-X-ray radiation (bremsstrahlung).

The objective of the radiation transport theory is to compute the readings of detector $J$ located in the field of radiation. The desired (measured) values are presented as the readings of some detector and are written as functional on the space of the transport equation solutions.

$$
J=\int Q(x) D(x) d x
$$

$D(x)$ is determined by the type of the desired (measured) value. We consider such registering facilities (detectors) whose readings $J$ are equal to the sum of the contributions of some particle's collisions in a sensitive volume of the detector (additive detectors). To evaluate the desired measured value by the Monte Carlo method, the random trajectories of the particles are simulated. The contributions of these trajectories to the detector's measurable value are summed up. The particle trajectory construction is performed according to the chosen physical model of the interaction between the radiation and matter.

The basic quantitative data of the transport model are the probability distributions of the particle's interactions with matter. These distributions are built by processing the cross sections (differential cross sections) of the corresponding interaction processes [8]. The main source of these data is the database of the National Centre of Nucleic Data (http://www.nndc.bnl.gov/sigma/).

\subsection{Model of the interaction of electrons with matter}

The following processes of the interaction of electrons with matter are considered:

- elastic scattering on atoms of matter leading to the deviation of an electron from its initial direction of motion;

- excitation of atoms accompanied by small losses of electron energy;

- ionization collisions or collision ionization (electro ionization) with the appearance of the secondary electron;

- electron slowing down in the Coulomb field of an atom results in generating the bremsstrahlung photon;

- electron-positron annihilation.

The generally accepted schemes of transport modelling are based on various modifications of the imbedded trajectories model (ETRAN [11], ITS3 [12], EGS4 [13], GEANT4 [14]). The electron scattering of electrons is usually described within the Goudsmit-Saunderson theory of multiple scattering [15]. Inelastic interactions of electrons with matter are described by various modifications of the approximation of continuous slowing down in most works. Fluctuations of the energy losses are accounted for by the Landau theory [16] using the Blunck and S. Leisegang corrections [17].

The implementation of these schemes for modelling electron transport on hybrid computing equipment is inefficient due to the complex internal logic of the schemes and the need to use iterative procedures in some cases. 
Simulating the trajectories of electrons carried out using the probability distributions of the characteristics of electrons [8] without the use of the mentioned widespread approximations in this work.

\subsection{Model of the interaction of photon radiation with matter}

Interaction model of gamma rays and $\mathrm{x}$-rays includes the following processes:

- coherent (Rayleigh) scattering;

- incoherent (Compton) scattering;

- photo absorption of quanta (photoionization of atoms);

- pair production.

Coherent scattering is the interaction process with a bound atomic electron without energy loss. The scattering results in the direction of the photon's movement changes. The cross section of this process is described by the Thompson formula subject to the relativistic form factor [18].

The Compton scattering of the photon on the free quiescent electron is described by the differential Klein-Nishina cross section and the electron connectedness in the atom is taken into consideration by the introduction of the scattering function [18].

The process of photoionization is the ionization of an atom by knocking the electron out of the atomic shell. An electron absorbs a photon and acquires its kinetic energy after subtracting the binding energy of an electron in an atom.

The interaction of a gamma-quant with an atom may lead to electron-positron pair production. The total cross section of this process is tabulated for instance, in [18]. Differential cross sections of the pair production are taken from $[14,19,20]$.

\section{THE PHOTON-ELECTRON CASCADE TRANSPORT MODELING}

An efficient algorithm for simulation of the cascade and its implementation require the solution of several problems:

- constructing the effective discrete geometrical model to minimize the cost of approximating complex objects;

- using the individual computational algorithms for each type of particle considering their physical properties;

- maximizing the statistical value of every trajectory for decreasing the computational cost.

\subsection{Geometrical model}

Modelling of radiation transport by use of the Monte Carlo method requires building huge number of particles random trajectories. Segments of intersection with different homogeneous parts of an object are all the information needed to construct trajectory chain from geometrical point of view. The interaction point and the type of the interaction are played along constructed set of the intervals by use of the probability distributions associated. The process keeps going until the particle "dies". Quasi-stationary gives possibility to get rid of grids of any kind. The most minimalistic and unified approach setting up geometrical model is to describe everything by surfaces. Parts of an object are described by closed bounding surfaces. The detection areas are either closed surfaces for volume-based measurements (density of energy deposit) or general surfaces for surface-based measurements (particles flux density). 
Areas of interest (see below) are described by its bounding surfaces as well.
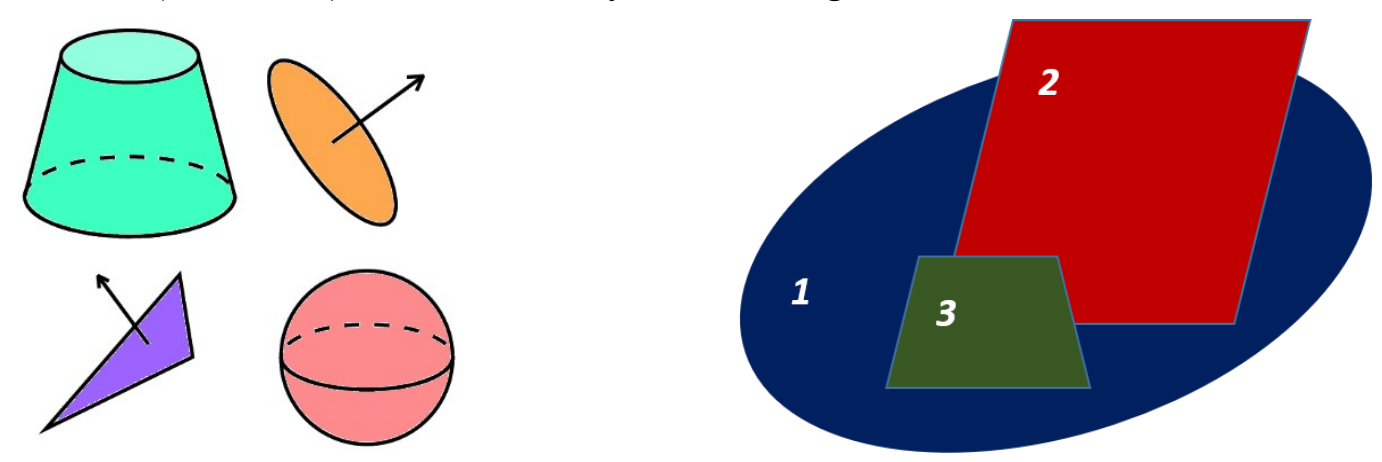

Fig. 1. Supported primitives - left; layers displacement - right

Surfaces consist of various types of "primitives". Currently supported are triangle, circle, sphere and frustum (Fig.1, left). The operation on the primitives are:

- getting coordinates of intersection between given ray along the particle motion direction and primitive;

- determining the distance from given point to primitive;

- testing the intersection between given line segment and the primitive.

Every surface is associated numbered "layer". It determines the molecular composition and density of a matter. The layers with larger numbers displace ones with smaller numbers if they occupy the same space (Fig. 1, right).

\subsection{Photons trajectories simulation}

Photons are characterized by relatively small number of collisions along trajectory and high penetrating ability. The construction of its trajectory includes object tracing and playing the interaction. The whole scheme is shown on fig. 2. It consists of the following steps:

- Intersections with the surfaces bounding the homogeneous components of the object are found. This is done by GPU;

- Intersection segments are formed using the points of the surface intersections;

- Interaction point is played on a set of intersection intervals (segments). The probability distributions of processes in corresponding material are loaded;

- Type of the interaction is played basing on loaded probability distributions. Photon properties are adjusted basing on played interaction. Additional particles that may appear due to interaction are stored for later simulation. 


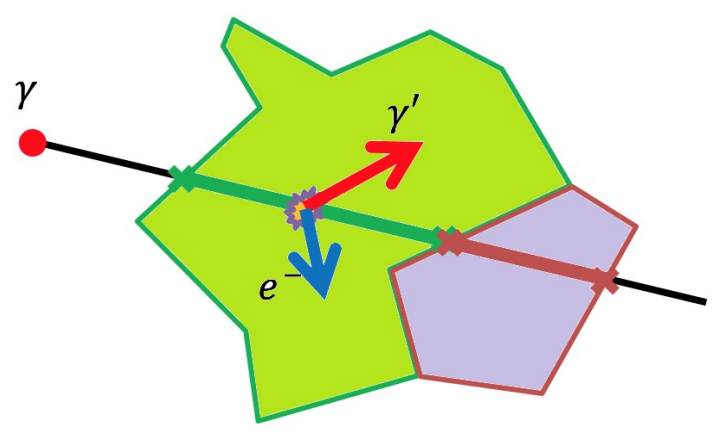

Fig. 2. Photon trajectory chain construction

The process repeats until trajectory ends. The approach is pretty much straightforward and efficient for this type of particle.

\subsection{Electron trajectories simulation}

Interactions between electron and matter are much different to photons. The number of collisions along trajectory is huge while penetrating ability is very low. The whole trajectory usually lays in small area inside an object. Straightforward approach used for photons is very excessive as full object tracing is barely needed at every step of the electron trajectory. Therefore, quasihomogeneous approach is used for electrons. Electron trajectory is built by large segments laying in homogeneous parts of an object. Those segments can contain hundreds to thousands of interactions. Even the whole trajectory. General scheme of electron trajectory segment construction is described below. Process starts with determining the layer in which the electron is located. Continuous slowdown approximation (CSDA) range of the electron in a current layer is calculated. CSDA range is a value showing the average length of electron trajectory until thermalization. The big circle on fig. 3 limits the area inside CSDA range of a particle. This is the area that particle will unlikely to leave. Defining the area that particle will likely to stay in allows ignoring the geometrical model outside this area for this electron. Surface elements outside CSDA range of a particle are filtered out - grayed out elements shown on fig. 3. Distance to the element from the original electron location is kept for elements that are inside of CSDA range. Elements are also sorted by this value ascending.
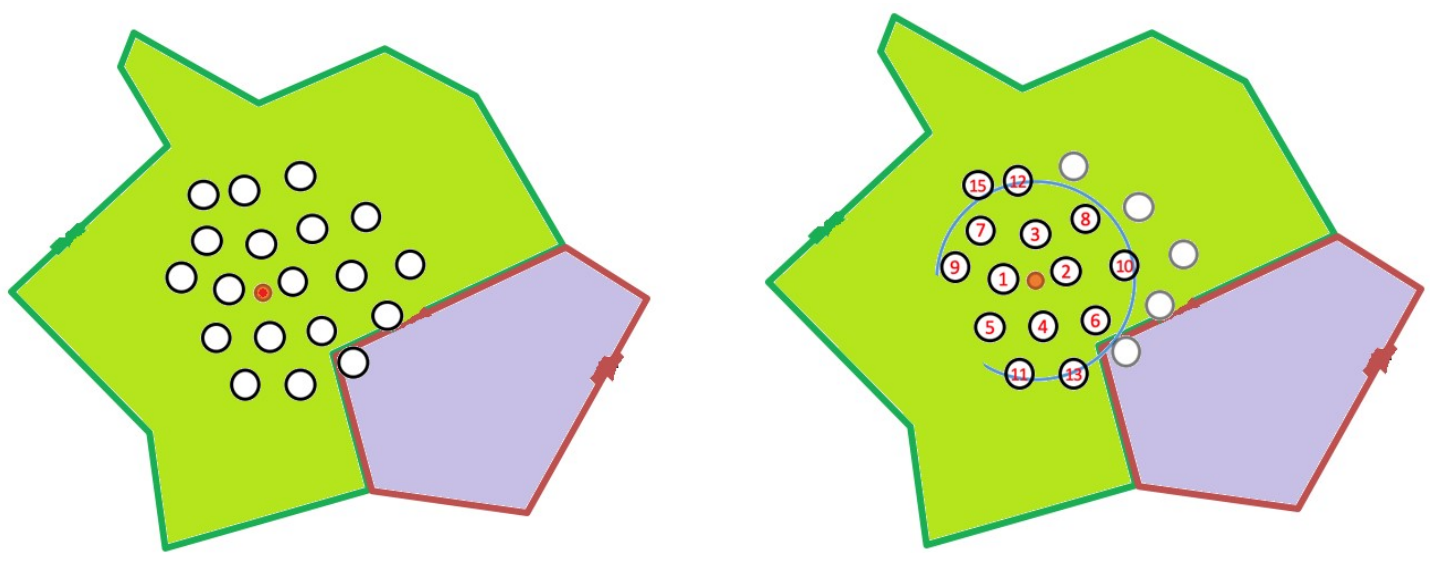
Fig. 3. Filtering out nearby primitives and sorting by distance

Trajectory segment construction is done very fast on GPU. Current distance $L$ to the original point where trajectory segment started is calculated every time particle position changes. Intersection checks are performed starting from closest elements to farthest and stops when distance to the next elements exceeds $L$. This keeps geometry checks minimal. On the right-side fig. 4 shows how determining the distance $L$ during trajectory construction allows to ignore even more of filtered (on the left side) elements.

Setting up very complex geometries and very high radiation energies may lead to situations when the number of the elements inside of CSDA range exceeds maximum. Filtering range is then decreased from CSDA range to something smaller that fits required number of elements. Decreasing filtering range increases the probability for particle to leave filtered area but keeps number of filtered elements relatively small.

The way trajectory intersection with an element is handled depends on the type of surface being intersected. Intersection with detector is simply recorded (position, direction, energy, weight etc.) to be processed on CPU later. Crossing the boundary of an object means that an electron can move to another layer. The CSDA range will change in this case. The trajectory segment construction ends at this point. Another stop-criteria for trajectory segment construction is reaching minimal energy supported by the software. Electron reaching this energy is considered thermalized. Last stop-criteria is electron leaving the area limited by CSDA range.
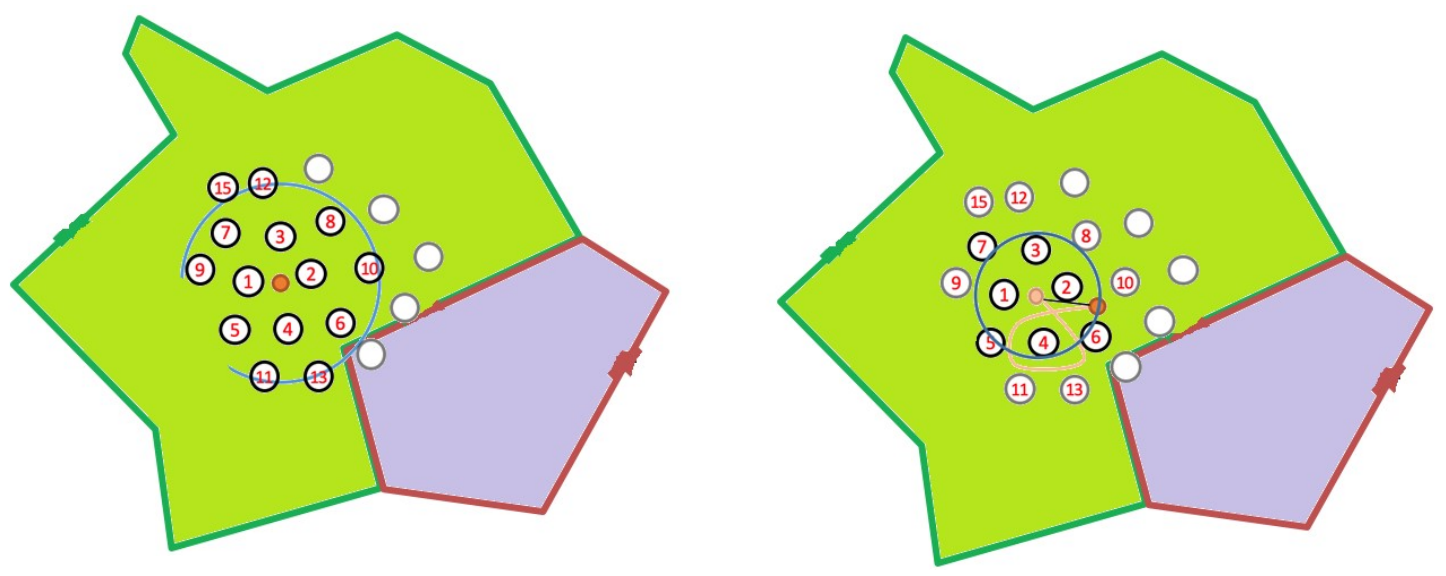

Fig. 4. Geometrical checks during electron trajectory simulation

Once trajectory segment construction ends all accumulated data is sent back to CPU. Data includes changed particle properties (position, direction, energy, weight etc.), detectors intersection history and newly born particles (impact ionization electrons and bremsstrahlung photons). These particles are saved for later modelling. Detector intersections are properly registered and whole process repeats for the electron.

\subsection{Algorithm for registration of improbable events}

Maximizing statistical value of the particle trajectory is very important when desired value 
is contributed by improbable events. Statistical evaluating the flux of emitting electrons from the surface of a plate irradiated by X-ray or evaluating the energy deposit in lightweight bremsstrahlung detector behind heavy target irradiated by electrons are both good examples of the problem.

Some portion of photons fly through the plate leaving no contribution in desired value at all in first example. The number of such photons depends on radiation energy, material and thickness of the plate. Problems of such a kind are usually solved by letting photon always interact with an object correspondingly decreasing its statistical weight. Unfortunately, only photons interacting in close proximity to surface can generate electron being able to emit. So, the most of modelled photon interactions will have zero statistical value.

In second example photon must interact with detector to contribute to energy deposit. Most of generated bremsstrahlung interacts with target rather than detector and gives no statistical contribution in detector.

General approach to solving the problems is developed. The main idea is introducing special "areas of interest" - volumes around detectors involving the electron registration (energy deposit, electron flux, current, charge). These volumes are described by bounding surfaces and naturally fit in developed geometrical model. Photon intersecting such area is split into several parts having corresponding statistical weights. Some of these parts interacts explicitly within area of interest. This allows electrons being explicitly born within proximity to detector significantly increasing statistical value of photon trajectory.

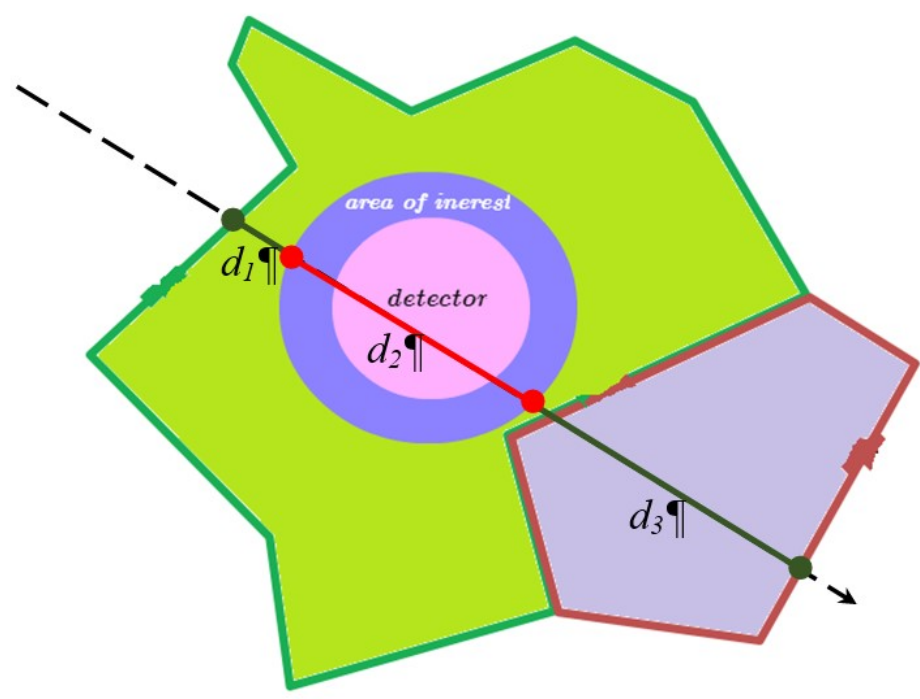

Fig. 5. Photons trajectory split

Fig. 5 shows an example of the photon trajectory intersecting area of interest inside an object. The photon trajectory inside the object consists of three intervals. First and third intervals are the outside the area of interest. Second one is intersection interval lays in the area of interest. The probabilities of photon to interact on these intervals are:

$$
P_{1}=1-e^{-\mu d_{1}} ; \quad P_{2}=e^{-\mu d_{1}} \cdot\left(1-e^{-\mu d_{2}}\right) ; \quad P_{3}=e^{-\mu\left(d_{1}+d_{2}\right)} \cdot\left(1-e^{-\mu d_{3}}\right),
$$


where $d_{1}, d_{2}$ and $d_{3}$ are lengths of intervals, $\mu$ full macroscopic cross section. In this case the trajectory is split in three parts (Fig. 5). Statistical weights are:

$$
w_{1}=w_{0} \cdot P_{1} ; \quad w_{2}=w_{0} \cdot P_{2} ; \quad w_{3}=w_{0} \cdot P_{3},
$$

where $w_{0}$ is initial weight of the photon. Thus, splitting trajectory allows electron to explicitly appear within area of interest. This electron will likely intersect detector contributing the statistical value. Trajectory splitting works the same way in case of intersections with multiple areas of interest.

\section{AN EXAMPLE OF COMPUTATION}

Interaction of radiation with matter is a cascade process. Radiation transport is accompanied by radiation-induced effects. Supercomputer simulation of radiation-induced charge effects in heterogeneous polydisperse materials is of interest in the investigation of their properties.

Some results of the modeling of the charge effects generated by X-ray radiation presented in this section.

Let us consider a fragment of a dispersed structure in the form of a cubic object with a side of 30 microns, in which polybutadiene is used as a binder, and an aluminum or dielectric ball with a diameter of 20 microns is an inclusion.

The fragment irradiated by photons of $20 \mathrm{keV}$ energy is depicted in Fig. 6. Red and blue balls are detectors for evaluating the charge density during simulation by Monte Carlo method.

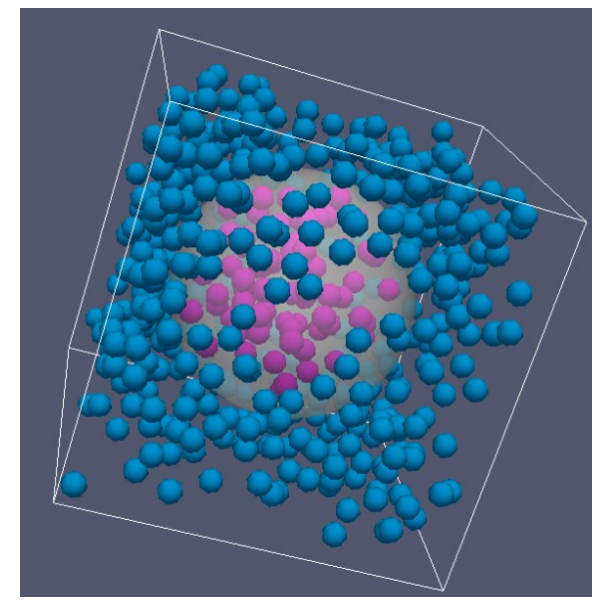

Fig. 6. Fragment of a dispersed structure with a detector system. Red balls are in the inclusion

The results of the calculation of the spatial distribution of the charge are presented below.

We note the common for both the inclusion regularities of the formation of radiationinduced charge effects. 


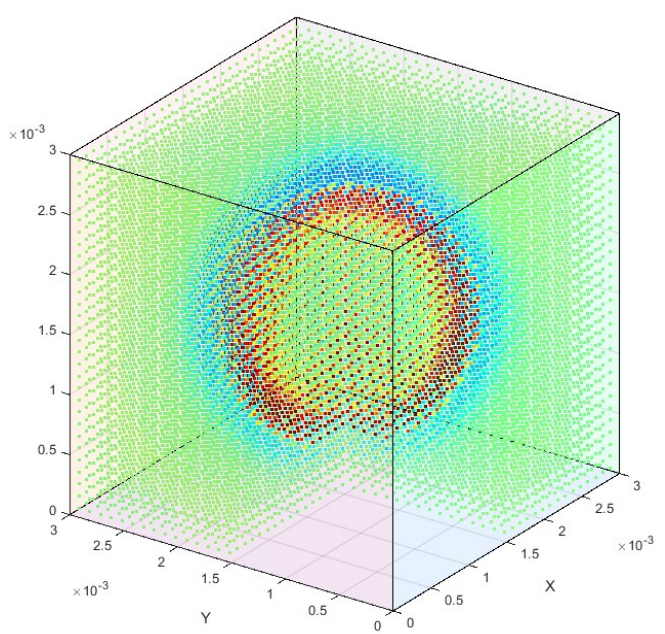

Fig. 7. 3D image of charge density distribution

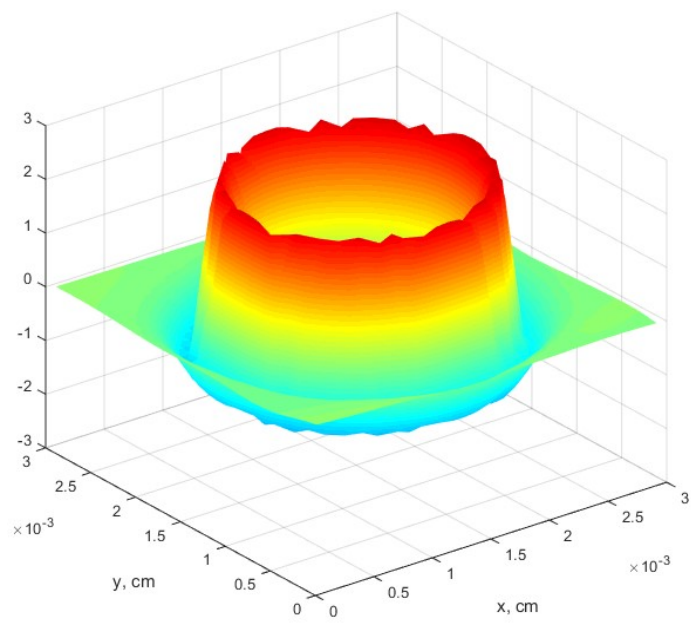

Fig. 8. Charge distribution in the plane perpendicular to the $\mathrm{z}$ axis through the center of inclusion

The Central inner part of the inclusion and the periphery of the binder are weakly charged due to the small path of electrons and, therefore, the local electronic equilibrium in these areas (Fig. 7, 8, green color).

A positive charge (red color) is formed in the inner boundary region of the inclusion due to the predominance of the electron departure from the inclusion to the binder over the reverse process. A negative charge (blue color) is formed in the binder near the inclusion due to the transfer of electrons from the inclusion into the binder.

The charge distribution along the straight line $x=y=15 \mathrm{mkm}$ is presented in Fig. 9,10. The boundaries of inclusions are marked with black dashed lines.

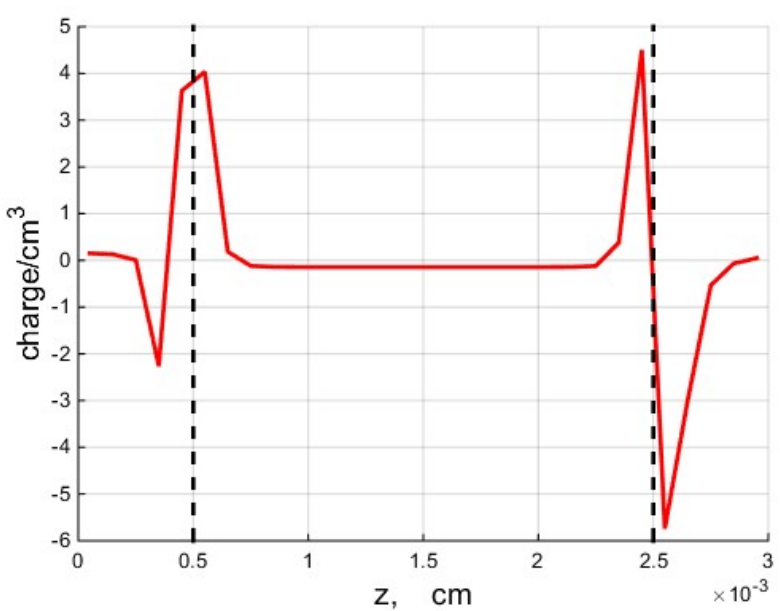

Fig. 9. The charge distribution in the fragment with aluminum inclusion

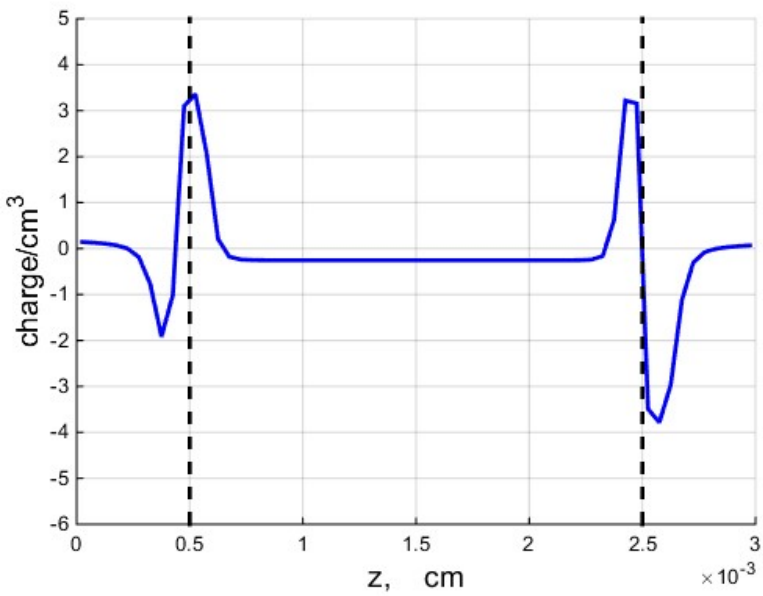

Fig. 10. The charge distribution in the fragment with dielectric inclusion

The graphs in Fig. 9,10 show sharply inhomogeneous spatial structure of the charge distribution generated by the interaction between radiation and dispersed structures. The 
inhomogeneities occur near the boundary surfaces of the binder and inclusions.

\section{CONCLUSION}

Effective statistical algorithms of the mathematical simulating the cascade processes of radiation transport have been developed by hybrid calculation procedures. These algorithms consider significant difference in physical properties of different particles and propose effective approaches to trajectory construction. Weight modifications of Monte-Carlo method significantly increasing the statistical value of random particle trajectories are built. Software is developed considering the features of the performed calculations on heterogeneous supercomputers by use of graphic accelerators as arithmetic co-processor. The performed model calculations illustrate the efficiency of the proposed method of modelling of cascade processes on computation facilities with a hybrid architecture.

Acknowledgements: This work is partially supported by Russian Fund for Basic Researches, grants N 17-01-00301 and N 18-01-00582.

\section{REFERENCES}

[1] Nerazrushaiushchii kontrol. Rossiia. 1990-2000, Spravochnik, V.V.Kliuev (Ed.), Mashinostroenie (2001).

[2] V. I. Mazhukin, V.V. Nosov. U. Semmler, "Issledovanie teplovykh i termouprugikh polei v poluprovodnikakh pri impulsnoi obrabotke” , Matematicheskoe modelirovanie, 12 (2), 75-83 (2000).

[3] K.K. Inozemtseva, M.B. Markov, F.N. Voronin, "The electromagnetic and thermomechanical effects of electron beam on the solid barrier", Mathematica Montisnigri, 39, 79-100 (2017).

[4] Ionizing radiation effects in MOS devices \& circuit, T.P. Ma and P.V. Dressendorfer (Eds.), John Wiley and Sons (1989).

[5] M.A. Trapeznikova, N.G. Churbanova and A.A. Lyupa, "Simulation of three-phase fluid flow in a porous medium with account of thermal effects", Mathematica Montisnigri, 33, 105-115 (2015).

[6] A.V. Utkin, "Analysis of parallelmolecular dynamics for MPI, CUDA and CUDA-MPI implementation", Mathematica Montisnigri, 39, 101-109 (2017).

[7] D.M. Ivashchenko, A.A. Fedorov, "Rossiiskie uskoriteli elektronov, ispolzuiushchiesia v kachestve modeliruiushchikh ustanovok", Voprosy atomnoi nauki i tekhniki, seriia "Fizika radiatsionnogo vozdeistviia na radioelektronnuiu apparaturu», 3, 120-128 (2002).

[8] M. E. Zhukovskiy, S. V. Podolyako, and R. V. Uskov, "Model of individual collisions for description of electron transport in matter," Math. Models Comput. Simul., 4 (1), 101-109 (2012).

[9] M. E. Zhukovskiy and R. V. Uskov, "Modelirovanie vzaimodeistviia gamma-izlucheniia s veshchestvom na gibridnykh kompiuterakh", Matematicheskoe modelirovanie, 23 (7), 20-32 (2011).

[10] M. E. Zhukovskiy and R. V. Uskov, "Matematicheskoe modelirovanie radiatsionnoi elektronnoi emissii na gibridnykh superkompiuterakh", Vychislitelnye Metody i Programmirovanie, 13 (1), 271$279(2012)$.

[11] https://link.springer.com/chapter/10.1007/978-1-4613-1059-4 7 (accessed October 22, 2019).

[12] J. A. Halbleib, R. P. Kensek, T. A. Mehlhom, G. D. Valdez, S. M. Seltzer and M. J. Berger, "ITS version 3.0: the integrated TIGER series of coupled electron/photon Monte Carlo transport codes", Report SAND91-1634, Sandia National Laboratories, Albuquerque, NM (1992).

[13] W. R. Nelson, Í. Hirayama and D. W. O. Rogers, "The EGS4 Code System", Report SLAC_265, Stanford Linear Accelerator Center, Stanford, CA (1985).

[14] http://geant4-userdoc.web.cern.ch/geant4userdoc/UsersGuides/PhysicsReferenceManual/html/index.html (accessed October 22, 2019). 
[15] S. Goudsmit and J. L. Saunderson, "Multiple scattering of electrons", Phys. Rev., 57, 24-29 (1940).

[16] L.D. Landau, "On the Energy Loss of Fast Particles by Ionization", J. Phys., 8 (4), 201-205 (1944).

[17] O. Blunck and S. Leisegang, "Zum Energieverlust schneller Elektronen in duennen Schichten", Zeitschrift für Physik, 128 (4), 500-505 (1950).

[18] https://www.nndc.bnl.gov/sigma/index.jsp?as=28\&lib=endfb7.1\&nsub=10010 (accessed October 22, 2019).

[19] https://oecd-nea.org/science/docs/2011/nsc-doc2011-5 (accessed October 22, 2019).

[20] https://mcnp.lanl.gov/ (accessed October 22, 2019).

[21] M. E. Zhukovskiy, S. V. Podolyako, and R. V. Uskov, "Modelirovanie perenosa elektronov v veshchestve na gibridnykh vychislitelnykh sistemakh", Vychislitelnye Metody i Programmirovanie, 12 (1), 152-159 (2011).

The part of results was presented at the thirteenth international seminar "Mathematical models \& modeling in laser-plasma processes \& advanced science technologies" (May 30 June 6, 2015, Petrovac, Montenegro).

Received August 12, 2019 